\title{
A Single Beam Smart Antenna for Wireless Communication in a Highly Reflective and Narrow Environment
}

\author{
K. Pirapaharan, Herman Kunsei, K.S. Senthilkumar, P.R.P Hoole, S.R.H. Hoole, Fellow, IEEE
}

\begin{abstract}
Multipath reflections are prevalent in underground mine wireless communication systems and are less constructive when an omnidirectional antenna is used. This phenomenon can be significantly controlled by eliminating the source of all multipaths with a single beam. The single beam must be rotatable towards the desired user to be of any use. The single directed beam will avoid generating multipath reflections and efficiently consume the valuable stored energy. In this paper we present an analysis of an array antenna using dipoles that forms a single beam without the need for reflectors or any complex arrangement of the array elements. It can be shown that dipole elements placed in a straight line are not effective in minimizing energy consumption and a minimum of three elements are sufficient for forming a single directed beam that is electronically rotatable to all directions. We have compared three, four and six elements for the accuracy. It is also shown that the elements of the array antenna should $b$ placed on the circumference of a circle to avoid re-computation of weights to rotate the beam on to any desired direction, thus significantly reducing the computational burden of the single beam, steerable smart antenna.
\end{abstract}

Index Terms-Smart Antenna, Phased Array, Undergound Mine, Undergound Communications.

\section{INTRODUCTION}

$\mathrm{T}$ he performance of wireless communications systems using an omnidirectional antenna [1-3] in underground mines is significantly degraded by multipath reflection phenomena among other impairments [4]. The undergound mine tunnel walls create a confinment that acts as a wave guide that allows the signal to reflect and thus propagate along the tunnel. The nature of signals propagating from an omnidirectional antenna creates multiple reflected signals along the tunnel which are detected a mobile user with varying arrival times [5] and phases. The varying signal characeristics increase the complexity of modelling the channel [6]. A dynamic channel requires the communications system to be adaptive.

Paper submitted for review on 20 June, 2016.

Prof. K. Pirapaharan and Herman Kunsei are with the Department of Electrical and Communications Engineering, Papua New Guinea University of Technology, Lae 411, PNG.

K.S. Senthilkumar is with St. George University, Grenada.

P.R.P. Hoole is with the Department of Electrical and Electronic Engineering, Faculty of Engineering, University Malaysia Sarawak, Sarawak, Malaysia.

S.R.H. Hoole (srhhoole@gmail.com), the corresponding author, is on the Election Commission, Sri Lanka, on leave from the Department of Electrical and Computer Engineering, Michigan State University.
Smart antennas have improved ground communications system and promise to be the better air interface for undergound communications challenges [7] to provide an effective and reliable communication system for use in normal and emergency circumstances $[8,9]$. To date most research in smart antennas has modeled array elements in a uniform linear array (ULA) $[10,11]$. Arranging dipoles in a ULA has the disadvantage of inefficient use of power and suffers from multuple part reflections when an image of the main beam is produced in the opposite direction on the central axis $[10,11]$. The drawback of such an antenna is more significant at the receiving end, where reflected waves will also be received and processed. This may degrade signal quality and lower system performance.

Therefore we propose a single beam smart antenna for underground communication systems that will nullify undesired reflected signals and efficiently use the limited power. With a single beam accepted at the receiving end, all other signals are nullified. Less efficient use of power was observed when the dipole elements were arranged in a linear or complex array [12]. Thus we analyzed a number of possible arrays with a sizeable number of elements in odd symmetrical geometry to form a steerable directed beam.

The results of this study with our models show that it is possible to have a more directed single beam with a minimum of three elements, and that as the number of elements is increased, the directivity and coverage range improve significantly. Finally, we demonstrate that the single rotating beam can scan a full $360^{\circ}$ area while nullifying all other beams outside the desired look angle.

\section{ADAPTIVE ARRAY MODEL}

Analytically we can show that any arbitrary set of dipoles arranged in a ULA would produce a radiation pattern that is symmetrical on both sides of the plane where the dipoles are placed [10]. As a result it is not possible to form a single beam and steer it in all directions by placing any number of dipoles in a straight line. However, our objective is to steer a single beam in the full $360^{\circ}$ azimuth angle range. Therefore, the three dipole elements should not be placed in a ULA. Consequently, we model a general setup as shown in Fig. 1 where dipoles are not placed in a straight line.

The respective complex current phasors of the dipoles are taken as $I_{1}, I_{2}$, and $I_{n}$. Hence the electric field (far-field) at the observation point $\mathrm{P}$ may be expressed as:

$$
E=A_{0} I_{1} e^{-j \beta r_{1}}+A_{0} I_{2} e^{-j \beta r_{2}}+\ldots+A_{0} I_{n} e^{-j \beta r_{n}}
$$

Where $A_{0}$ and $\beta$ are a constant and the phase constant, respectively. 